\title{
CNS Progenitor Cells Promote a Permissive Environment for Neurite Outgrowth via a Matrix Metalloproteinase-2- Dependent Mechanism
}

\author{
Yiqin Zhang, ${ }^{1}$ Henry J. Klassen, ${ }^{2}$ Budd A. Tucker, ${ }^{1}$ Maria-Thereza R. Perez, ${ }^{3}$ and Michael J. Young ${ }^{1}$ \\ ${ }^{1}$ Schepens Eye Research Institute, Department of Ophthalmology, Harvard Medical School, Boston, Massachusetts 02114, ${ }^{2}$ Stem Cell Research, Children's \\ Hospital of Orange Country, Orange, California 92868, and ${ }^{3}$ Wallenberg Retina Center, Department of Ophthalmology, Lund University, SE-22100 Lund, \\ Sweden
}

Transplantation of progenitor cells to the CNS has shown promise in neuronal and glial replacement and as a means of rescuing host neurons from apoptosis. Here we examined the effect of progenitor grafts on neurite extension in the degenerating retina of $r d 1$ (retinal degeneration 1) mice. Transplantation of retinal progenitor cells induced increased matrix metalloproteinase-2 (MMP2) secretion, partly from activated glial cells, which was then activated by neuronally expressed MMP14. Active MMP2 resulted in proteolysis of the neurite outgrowth inhibitors $\mathrm{CD} 44$ and neurocan in the degenerative retina, allowing significantly increased neurite outgrowth across the border between abutting nondystrophic and $r d 1$ retinas. Progenitor-induced enhancement of outgrowth was abrogated by an MMP inhibitor or by coculture with retinal explants from $M M P 2^{-1-}$ mice. This study provides the first identification of an MMP2-dependent mechanism by which exogenous progenitor cells alter the host environment to promote neural regeneration. This suggests a novel therapeutic role for progenitor cells in the treatment of CNS degenerative diseases.

Key words: retina; progenitor cell; cell transplantation; cell migration; neurite outgrowth; MMP-2

\section{Introduction}

The regenerative capacity of the adult mammalian CNS is highly limited with respect to both the generation of new neurons and reconstruction of synaptic pathways. Neurons in the injured adult CNS rarely succeed in regrowing to their targets and almost never form new functional synapses. Although adult neurons have a reduced capacity for regeneration, even grafted embryonic neurons exhibit a limited ability to extend axons into denervated targets in the adult CNS (Gaillard et al., 1998; Aramant et al., 1999; Kwan et al., 1999; Zhang et al., 1999; Ghosh et al., 2004). It is now well established that the environment of the mature mammalian CNS is not conducive to axonal regrowth and that the nonpermissive nature of this environment is strongly linked to the presence of inhibitory molecules. After injury or disease, reactive gliosis and glial scar formation, together with upregulation of neurite growth inhibitors, present major barriers to neurite outgrowth and the reestablishment of functional relay pathways (Asher et al., 2000; Bradbury et al., 2002).

Recent studies show that the amelioration of functional defi-

\footnotetext{
Received Jan. 17, 2006; accepted Feb. 10, 2007.

This work was supported by National Eye Institute Grant 09595 (M.J.Y.), Siegal Foundation, Department of Defense, Natural Sciences and Engineering Research Council of Canada, and Hoag Foundation.

Correspondence should be addressed to Michael J. Young, Schepens Eye Research Institute, Department of Ophthalmology, Harvard Medical School, 20 Staniford Street, Boston, MA 02114. E-mail: mikey@vision.eri.harvard.edu.

H. J. Klassen's present address: Department of Ophthalmology, University of California, Irvine, School of Medicine, Orange, CA 92868

DOI:10.1523/JNEUROSCI.0200-07.2007

Copyright $\odot 2007$ Society for Neuroscience $\quad$ 0270-6474/07/274499-08\$15.00/0
}

cits observed after neural progenitor cell (NPC) transplantation frequently occurs even when the grafted cells remain primarily undifferentiated (Ogawa et al., 2002; Teng et al., 2002). Functional recovery in this setting may result from the promotion of host regeneration rather than cell replacement, possibly through NPC-mediated alteration of the inhibitory environment of the host. These findings point to a novel potential application of progenitor cells in CNS repair and provide additional avenues for the development of cell-based therapies in neurodegenerative disorders. At present, we have a very limited understanding of the molecular mechanisms underlying the interactions of grafted progenitor cells with the host microenvironment. Although previous studies have demonstrated a trophic role for NPCs that may enhance neurite growth ( $\mathrm{Lu}$ et al., 2003), very little is known about the potential of these cells to regulate expression of inhibitory cues in the adult CNS.

Matrix metalloproteinases (MMPs) are key processing enzymes that modulate cell behavior through the cleavage of a range of bioactive molecules. These include extracellular matrix (ECM) and cell surface molecules, many of which exhibit profound growth-inhibitory properties (Ferguson and Muir, 2000; McCawley and Matrisian, 2001; Costa et al., 2002). For example, MMP2 is involved in the regulation of cell motility and neurite outgrowth via the processing of inhibitory chondroitin sulfate proteoglycans (CSPGs) that are upregulated in the CNS after injury or degeneration (Ferguson and Muir, 2000). We demonstrated previously that neurocan (a CSPG) and CD44 (a glycoprotein) are present at the outer surface of the retina and may 
limit extension of neurites from a subretinal graft into the host retina (Zhang et al., 2004). Here, we report a new type of neurite outgrowth-promoting activity associated with exogenous CNS progenitor cells and mediated by induction of MMP2 expression in Müller cells with subsequent proteolysis of the neurite outgrowth inhibitors neurocan and CD44.

\section{Materials and Methods}

Animals. Postnatal day 1 (P1) mice expressing the enhanced variant of green fluorescent protein (GFP) (gift from Dr. Okabe, University of Osaka, Osaka, Japan) or B6 mice (The Jackson Laboratory, Bar Harbor, $\mathrm{ME)}$ not expressing this protein were used for isolation of retinal precursor cells (RPCs) and NPCs. Transgenic mice expressing enhanced cyan fluorescent protein (CFP) (The Jackson Laboratory) at the age of P0 were used as donors for retina transplantation. Retinal degenerative mice (C3H/HeJ; The Jackson Laboratory), 60-90 d old, were used as recipients. In the $r d 1$ (retinal degeneration 1) retina, near complete loss of rod photoreceptors has occurred by the third postnatal week. CFP, $r d 1$, B6, or $M M P 2^{-1-}$ mice provided the abutting retinal tissue explants used in culture. The experiments were conducted with the approval of the Schepens Eye Research Institute Animal Care and Use Committee and the Association for Research in Vision and Ophthalmology Statement for the Use of Animals in Ophthalmic and Vision Research.

Preparation of RPCs and NPCs. Eyes and whole brains were removed and placed in HBSS. Neural retinas were carefully dissected away from the optic disc and ciliary marginal zone. Retinal and brain tissues were processed separately. Each was minced and digested in collagenase $(0.1 \%$ in PBS, pH 7.2-7.4; Sigma, St. Louis, MO) at room temperature. Liberated cells were collected through a $100 \mu \mathrm{m}$ mesh strainer, centrifuged, and then resuspended in neurobasal medium supplemented with 20 $\mathrm{ng} / \mathrm{ml}$ epidermal growth factor, $2 \% \mathrm{~B}-27$ supplement (Invitrogen, Carlsbad, CA), $100 \mu \mathrm{g} / \mathrm{ml}$ penicillin/streptomycin, $2 \mathrm{~mm}$ L-glutamine, and $2000 \mathrm{U}$ of nystatin (Sigma). Cells were transferred to a six-well plate and cultured at $37^{\circ} \mathrm{C}$ in a $5 \% \mathrm{CO}_{2}$ incubator. Fresh medium $(0.5 \mathrm{ml})$ was added to each well every $2 \mathrm{~d}$. Primary neurospheres formed within the first week in culture. These proliferating cultures were passaged weekly for 12 weeks, at which point they were used for the experiments described here.

Transplantation. The transplantation procedure used here was described previously (Zhang et al., 2004). Briefly, donor retinas were dissected free from the retinal pigmented epithelium and optic nerve head and then cut into small fragments in serum-free medium [DMEM/ Ham's F-12 (Omega Scientific, Tarzana, CA) supplemented with $2 \mathrm{~mm}$ L-glutamine and $100 \mu \mathrm{g} / \mathrm{ml}$ penicillin/streptomycin (Sigma)]. Recipient mice were anesthetized by an intraperitoneal injection of Rompun (100 $\mathrm{mg} / \mathrm{kg})$ and Ketalar $(100 \mathrm{mg} / \mathrm{kg})$. Donor retinal tissue $(0.3 \mu \mathrm{l}$; P0 neural retinal fragments) and/or RPC spheres $(0.3 \mu \mathrm{l} ; \sim 50 \mu \mathrm{m}$ in diameter $)$ were subsequently taken up into a plastic pipette tip and gradually injected through the sclera into the subretinal space of $r d 1$ mice. Animals were killed $30 \mathrm{~d}$ after surgery. No immunosuppression was used.

Explant cultures. The procedure used for abutting-retina cultures has been described previously (Zhang et al., 2003). Briefly, retinas were dissected, transferred to freshly prepared HBSS (Invitrogen), and cut into four pieces. Topographically equivalent pieces from two different explants (CFP and $r d 1$ ) were then selected. RPC or NPC spheres (4-10 spheres, $\sim 50-400 \mu \mathrm{m}$ in diameter) were placed on the inner (vitreal) surface of the CFP-derived retinal explant. The $r d 1$-derived retinal explant was then placed so as to partially cover the RPCs/NPCs, as well as the underlying CFP-derived explant, with the outer plexiform layer facing the ganglion cell layer of the CFP-derived explant. Explant pairs without added progenitor cells served as controls. All explant pairs were mounted onto Millicell-CM organotypic tissue culture plate inserts (Millipore, Bedford, MA), with the CFP mouse-derived explant closest to the filter, and incubated in the serum-free medium described above at $37^{\circ} \mathrm{C}$ for $7 \mathrm{~d}$, with media changes every $2 \mathrm{~d}$.

$D L-\alpha$-Aminoadipic acid treatment. To suppress glial cell activity, a series of experiments were performed in which the explant/RPC coculture system was plated in normal media supplemented with $0.5 \mathrm{M}$ of the glial cell toxin DL- $\alpha$-aminoadipic acid (Sigma). As indicated above, these explant cultures were allowed to survive for $7 \mathrm{~d}$ with media changes at days 2, 4, and 6. Explants were subsequently harvested, cryosectioned, and processed for immunostaining.

Recombinant MMP2 activation and treatment. Inactive, pro-MMP2 (2 $\mu \mathrm{g} / \mathrm{ml}$; Calbiochem, San Diego, CA) was activated before explant treatment by incubating the enzyme with $1 \mathrm{~mm} p$-aminophenylmercuric acetate $\left(2: 1\right.$; Sigma) for $2 \mathrm{~h}$ at $37^{\circ} \mathrm{C}$. Activated MMP2 was subsequently added to explant cultures when appropriate at $100 \mathrm{ng} / \mathrm{ml}$, for $12 \mathrm{~h}$, at $37^{\circ} \mathrm{C}$. After treatment, explants were harvested, cryosectioned, and immunostained.

Histology and immunocytochemistry. Tissue fixation, sectioning and immunocytochemistry (ICC) were performed as described previously (Zhang et al., 2003). The primary antibodies used were raised against GFP (1:100; Sigma), CD44 (1:100; Sigma), neurocan (1:500; Renee K. Margolis, Department of Pharmacology, State University of New York, Brooklyn, NY), MMP2 (1:50; Chemicon, Temecula, CA), MMP14 (1: 100; Chemicon), PKC (1:1000; Santa Cruz Biotechnology, Santa Cruz, CA), nestin (1:1; Developmental Studies Hybridoma Bank, University of Iowa, Iowa City, IA), neurofilament 200 (1:1000; Sigma), calbindin (D-28K, 1:800; Sigma), calretinin (1:100; Chemicon), rhodopsin (2D4, 1:500; gift from Dr. Robert Molday, University of British Columbia, Vancouver, British Columbia, Canada; 1:500), recoverin (1:200; Chemicon), cellular retinaldehyde-binding protein (1:500; Chemicon), and GFAP (1:400; Invitrogen). The secondary antibodies were cyanine 3 (Cy3)-conjugated goat anti-mouse and $\mathrm{Cy} 3$-conjugated goat anti-rabbit (1:800; Jackson ImmunoResearch, West Grove, PA). Sections were viewed using a light microscope equipped for fluorescence microscopy.

In situ zymography. Eyes containing transplants were quickly removed without fixation and frozen on dry ice. After cryostat sectioning, the specimens were incubated overnight at $37^{\circ} \mathrm{C}$ in fluorescent-labeled gelatin from Invitrogen (according to the instructions of the manufacturer). In this method, proteolytic digestion of the substrate results in unblocking of green fluorescence. Some sections were subsequently fixed in $4 \%$ paraformaldehyde and subjected to double labeling using antibodies selected from those described above.

Gel zymography. Conditioned medium containing equal amounts of protein was loaded onto a $10 \%$ SDS-polyacrylamide gel containing gelatin (Bio-Rad, Hercules, CA). After electrophoresis, gels were washed in $5 \%$ Triton X-100 and then incubated in MMP assay buffer (Bio-Rad). Bands were visualized with Coomassie Brilliant Blue and destained in $30 \%$ methanol and $10 \%$ acetic acid.

Western blot. Retinas were homogenized in lysis buffer (50 mм Tris$\mathrm{HCl}, \mathrm{pH}$ 7.6, $150 \mathrm{~mm} \mathrm{NaCl}, 10 \mathrm{~mm} \mathrm{CaCl}_{2}, 1 \%$ Triton X-100, and $0.02 \%$ $\mathrm{NaN}_{3}$ ). Some of the samples were then treated with $0.02 \mathrm{U}$ of chondroitinase $\mathrm{ABC}$ (protease-free; Roche, Indianapolis, IN) for $3 \mathrm{~h}$ at $37^{\circ} \mathrm{C}$. Conditioned medium or extracted protein was subjected to SDS-PAGE under nonreducing or reducing conditions and transferred to a nitrocellulose membrane (Bio-Rad). The membrane was blotted with the antibodies specific to neurocan and CD44S (1:100; Chemicon).

Immunoelectron microscopy. Retinas that had received transplants were processed using pre-embedding immunocytochemistry as described previously (del Rio and DeFelipe, 1997). Briefly, after preincubation in $3 \%$ normal goat serum in PBS, specimens were incubated in rabbit anti-GFP (1:60; Sigma) for $48 \mathrm{~h}$ at $4^{\circ} \mathrm{C}$ and processed with avidinbiotin-peroxidase using a Vectastain $\mathrm{ABC}$ immunoperoxidase kit, as per instructions. Specimens were next reacted with $0.04 \%$ 3,3diaminobenzidine tetrahydrochloride, followed by incubation with the same reagent containing $0.005 \% \mathrm{H}_{2} \mathrm{O}_{2}$, and postfixed with $2 \%$ paraformaldehyde $/ 2.5 \%$ glutaraldehyde overnight at $4^{\circ} \mathrm{C}$. Specimens were then osmicated, dehydrated, and embedded in Epon-Araldite for sectioning. The ultrathin sections $(80 \mathrm{~nm})$ generated were stained with uranyl acetate and lead citrate and viewed and photographed with a transmission electron microscope (EM410; Philips, Aachen, Germany).

Quantification. Fibers expressing neuronal markers, originating from CFP-derived retinas or from $r d 1$-derived retinas and crossing the interface of the abutting retinas, were counted in every fifth serial section (12 $\mu \mathrm{m}$ thick) from each specimen. The area of the CFP-derived retina adjacent to $r d 1$ explants was also measured. The ratio of the number of 
fibers to the abutting area were calculated and expressed as the number per $100 \mu \mathrm{m}$. Statistical analysis was performed with an unpaired $t$ test.

\section{Results}

\section{Engrafted RPCs enhance expression and activation of} host MMP2

To examine whether CNS progenitor cells alter MMP2 expression and activity in $r d 1$ retina, RPCs were transplanted to the subretinal space of 9-week-old $r d 1$ mice, followed by ICC and in situ gelatinase zymography. The antibody used for ICC specifically detects pro-MMP2, whereas in situ gelatinase zymography detects active gelatinase but cannot distinguish between different gelatinase subtypes (e.g., MMP2 and MMP9). We also used a different technique known as gel gelatinase zymography, which can distinguish between active MMP2 and MMP9.

One month after transplantation, a large number of RPCs had migrated into the $r d 1$ retina (Fig. $1 A$ ) in association with increased retinal pro-MMP2 immunoreactivity and gelatinase activity (Fig. $1 A, B$ ) compared with controls, which received grafts of solid neural retina (Fig. $1 C, D$ ). Increased pro-MMP2 expression was restricted to areas of the host retina in which donor cells had migrated and localized in radial processes characteristic of Müller glia cells (Fig. 1A). NPCs had an effect similar to RPCs (data not shown), and neither type of grafted progenitor cell expressed pro-MMP2 (Fig. $1 A, C$ ).

The transplantation results were replicated in vitro using $r d 1$ explants cocultured with RPCs, thus allowing pharmacological manipulation of the experimental conditions. Selective suppression of Müller cell reactivity was obtained by addition of DL- $\alpha$ aminoadipic acid to the culture media (Jablonski and Iannaccone, 2000). This treatment eliminated increased pro-MMP2 immunoreactivity in cocultured $r d 1$ retinas (Fig. $1 E$ ) compared with control (Fig. $1 F$ ), further supporting a role for reactive Müller cells in MMP2 induction.

In situ zymography demonstrated that active gelatinase was present throughout the $r d 1$ retina, although labeling was more intense in the outermost layer (corresponding to the inner nuclear layer of the degenerated $r d 1$ retina) at 1 month after transplantation (Fig. $1 B$ ). By combining in situ zymography with immunostaining for retinal cell markers, we identified cell types that colocalized with active gelatinase. The majority of these cells were neurons, some of which coexpressed neuronal markers (PKC in Fig. $1 G)$, whereas others were Müller cells or astrocytes that expressed GFAP (Fig. $1 \mathrm{~B}$ ). Interestingly, migrating GFP-positive $\left(\mathrm{GFP}^{+}\right)$progenitor cells grafted to $r d 1$ retinas did not express detectible levels of pro-MMP2. The majority of cells in $r d 1$ retinas positive for active gelatinase coexpressed MMP14 (Fig. 1G), a membrane-type MMP that binds to and activates pro-MMP2 (Overall and Lopez-Otin, 2002). Triple labeling with in situ zymography, MMP14, and neuronal markers, together with constitutive GFP expression, identified the majority of active gelatinase-expressing cells that coexpressed MMP14 as host neurons (Fig. $1 G$ ). Some grafted progenitor cells in $r d 1$ retina also expressed MMP14 (data not shown). Gel zymographic analysis of conditioned media as well as cell lysates and membrane fractions further confirmed that the progenitor cells did not secrete detectible levels of MMP2 or MMP9, yet these progenitor cells greatly enhanced the secretion and activation of MMP2 in cocultured $r d 1$ retinal explants (Fig. $1 H$ ).

We then asked whether MMP2 induction is related to progenitor cell migration, because the induction was seen in the areas in which grafted cells were present. To evaluate this issue, we grafted two types of cellular preparations known to exhibit limited mi-
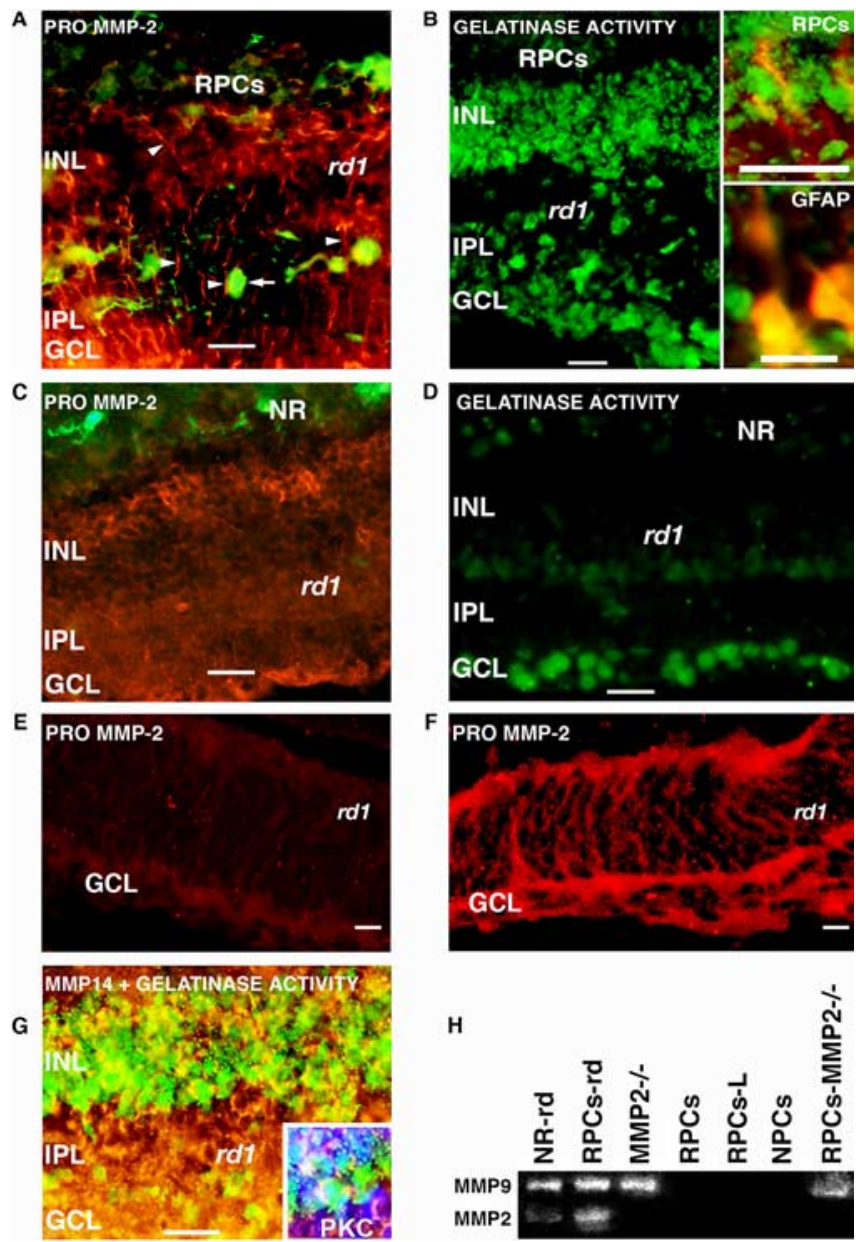

Figure 1. MMP2 expression and activation in $r d 1$ retinas 1 month after transplantation. $\boldsymbol{A}$, Upregulation of pro-MMP2 immunostaining is seen in radial Müller cell processes (red processes, identified by arrowheads) after RPC engraftment. Enhanced immunoreactivity was located in regions in which RPCs (green cell, identified by arrow) had migrated into the host retina. $\boldsymbol{B}$, In situ gelatinase zymography shows markedly increased gelatinase activity (green) in most layers of the $r d 1$ retina after engraftment of RPCs. Insets show colocalization (yellow) of double labeling with zymography (green) and immunostaining (red) against, GFP and GFAP.C, Pro-MMP2 immunostaining (red) in controls: diffuse immunoreactivity is present throughout the retina after engraftment with neural retinal tissue (NR; green). D, In situ gelatinase zymography in controls: gelatinolytic activity (green) is low throughout the inner retina, with moderate activity in the $\mathrm{GCL}$ after engraftment with neural retinal tissue. $\boldsymbol{E}, \boldsymbol{F}$, Pro-MMP2 immunostaining in DL- $\alpha$-aminoadipic acid-treated $(\boldsymbol{E})$ and untreated $(\boldsymbol{F})$ cocultures: normal increases seen in pro-MMP2 levels after coculture with RPCs is inhibited with DL- $\alpha$-aminoadipic acid treatment. G, Double labeling of MMP14 immunostaining (red) with gelatinase zymography (green) shows colocalization (yellow) of MMP14 immunoreactivity and gelatinolytic activity in the inner nuclear layer (INL) and on some cells in the GCL. Inset shows colocalization (white) of triple labeling for MMP14 (red), PKC $\alpha$ (blue), and gelatinolytic activity (green). $\boldsymbol{H}$, Gel gelatinase zymography of conditioned media shows increased MMP2 gelatinolytic activity in rd1 retinas cocultured with RPCs (RPCs-rd) compared with controls of neural retinal tissue cocultured with $r d 1$ retinas (NR-rd). Neither MMP2 nor MMP9 gelatinolytic activity was detected from either conditioned media from RPCs or NPCs alone or from RPC lysates or membrane fractions (RPCs-M). MMP9, but not MMP2, gelatinolytic activity was detected from MMP2 knock-out $\left(M M P 2^{-/-}\right)$retinas as well as from $M M P 2^{-/-}$retinas cocultured with RPCs (RPCs$\mathrm{MMP2}^{-/-}$). Scale bars, $30 \mu \mathrm{m}$. IPL, Inner plexiform layer.

gratory behavior, neonatal full thickness retina and large $(>200$ $\mu \mathrm{m}$ in diameter) RPC spheres. Neither elicited increased expression or activation of MMP2 in the host retina (neural retina graft in Fig. $1 C, D$ ), indicating that induction of MMP2 requires migration of progenitor cells into the tissue. 

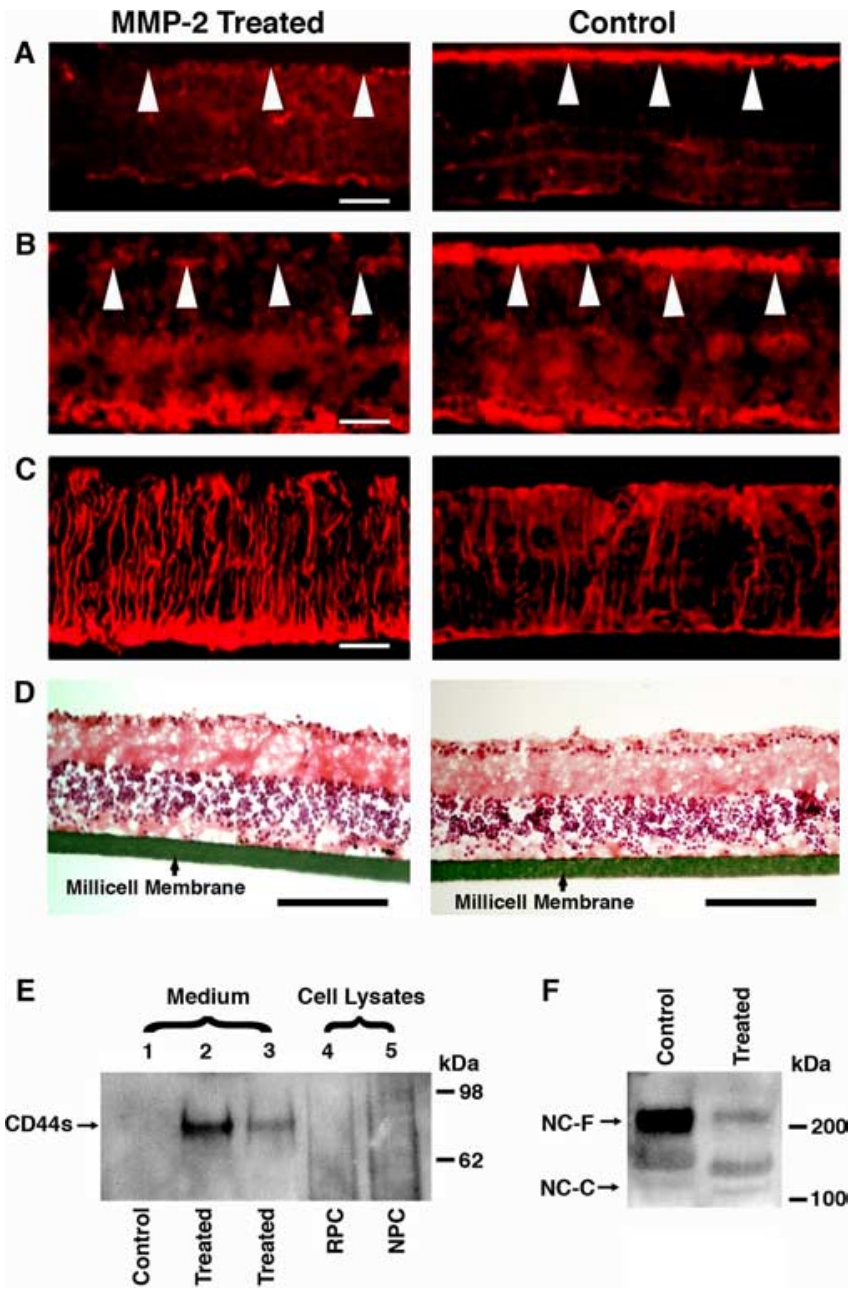

Figure 2. Degradation of CD44 and neurocan by MMP2 in vitro. $\boldsymbol{A}-\boldsymbol{D}$, Incubation of $r d 1$ retinas with recombinant MMP2 for $12 \mathrm{~h}$ led to a decrease in $C D 44(\boldsymbol{A})$ and neurocan $(\boldsymbol{B})$ immunoreactivity on the outer surface of $r d 7$ retinas (arrowheads) compared with controls. However, the treatment did not appreciably change GFAP immunoreactivity in reactive Müller cells $(\boldsymbol{C})$ or significantly alter retinal morphology (D). $\boldsymbol{E}, \boldsymbol{F}$, Western blot shows $\mathrm{CD} 44(\boldsymbol{E})$ and neurocan $(\boldsymbol{F})$ cleavage products in $r d 1$ explants treated with conditioned medium from $r d 1$ retina/RPC cocultures. Lanes 1-3, Protein was extracted in culture medium from rd1 explants treated with conditioned medium from RPC (lane 1), rd1 retina/RPC cocultures (lane 2), or $r d 1$ retina/NPC cocultures (lane 3). Lanes 4, 5, Protein was extracted from RPC (lane 4) or NPC lysate (lane 5). Scale bars: $A-C, 30 \mu \mathrm{m} ; \boldsymbol{D}, 200 \mu \mathrm{m}$.

\section{Active MMP2 degrades neurocan and CD44}

CD44 and neurocan immunostaining was evident as a continuous band adjacent to the outer surface of the $r d 1$ retina. It has been suggested previously that this zone poses a barrier to the extension of neurites from subretinal tissue grafts into the dystrophic retina (Zhang et al., 2004). MMP2 can specifically process neurocan in vitro (Turk et al., 2001), but whether it can also degrade CD44 is unknown. Moreover, it is unclear whether MMP2 can efficiently degrade either neurocan or CD44 in the setting of retinal degeneration. To address these questions, we treated $r d 1$ explants with preactivated recombinant MMP2 and examined whether this had an effect on CD44 and neurocan expression using ICC and Western blot analysis. The $r d 1$ explants were placed with the outer retina adjacent to the membrane, so that the outer retinal surface was in direct contact with the medium containing recombinant MMP2. After $12 \mathrm{~h}$ of treatment, immunoreactivity for both neurocan and CD44 was essentially abolished. This was particularly evident at the outer surface of the
RPCs-rd1

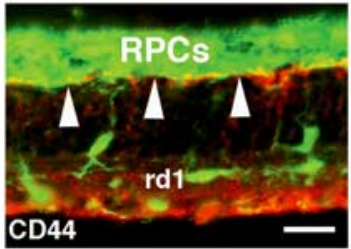

B

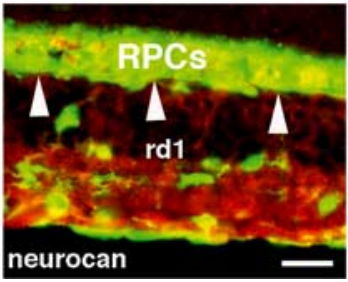

NR-rd1
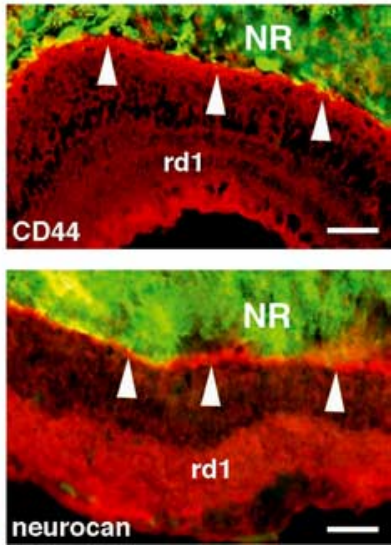

Figure 3. Reduction of $\mathrm{CD} 44$ and neurocan in $r d 7$ retinas after engraftment with progenitor cells in vivo. CD44 (A) and neurocan (B) immunoreactivity was greatly reduced (arrowheads in left column) along the interface of graft and host in regions in which RPCs had migrated into the host retina at $30 \mathrm{~d}$ after transplantation (left column) compared with controls in which $r d 1$ retinas were engrafted with neural retinal tissue (NR-rd1; right column). Scale bars, $30 \mu \mathrm{m}$.

degenerative retina in which levels of these molecules would be highest (Fig. 2A,B). Although this result is consistent with MMP2-mediated degradation of extracellular proteins, to ensure that the result was not attributable to an MMP2-related cytotoxic effect on neurocan- and CD44-producing cells, we performed immunocytochemical analysis for GFAP expression and hematoxylin and eosin staining on treated and untreated retina. The results showed that Müller cells expressed high levels of GFAP, indicating that these cells were present and viable after MMP2 treatment (Fig. 2C) and that no significant morphological differences exist between treated and control retinas (Fig. 2D). We then examined whether conditioned medium from $r d 1$ retina/ progenitor cell cocultures (hence containing active MMP2) was also capable of degrading neurocan and CD44 in the $r d 1$ retina. Western blot results demonstrated that treatment of $r d 1$ explants with conditioned medium led to the presence of $70 \mathrm{kDa}$ CD44 isoform in the culture medium (Fig. 2E) as well as a decrease in $220 \mathrm{kDa}$ full-length neurocan with a concomitant increase of the $150 \mathrm{kDa}$ C-terminal fragment in retinal lysates (Fig. $2 \mathrm{~F}$ ). Thus, MMP2 and conditioned medium from $r d 1$ retinal progenitor cell cocultures can degrade neurocan and CD44. In vivo experiments confirmed that engraftment of RPCs in the $r d 1$ retina promoted the induction of MMP2 and significantly reduced neurocan and CD44 immunoreactivity compared with controls (Fig. $3 A, B$ ).

\section{Engrafted RPCs promote neurite extension and} neural connectivity

Next, we cotransplanted neural retina (harvested at P0) and RPCs or NPCs to the subretinal space of $r d 1$ mice ( 8 weeks of age) and observed the extension of neurites from the graft into the host retina as well as from the host into the graft. In addition, we used an abutting retinal explant coculture system, in which a nondystrophic neural retina ( $\mathrm{P} 0)$ is placed on the outer surface of a degenerated $r d 1$ retina, with progenitor cells added at the interface of the two retinas. This coculture system simulates full thickness retinal transplantation and is useful for examining neuronal plasticity and modeling connectivity between graft and host (Zhang et al., 2003, 2004).

In both transplantation and explant models, it is important to be able to distinguish the various components from one another, 
A

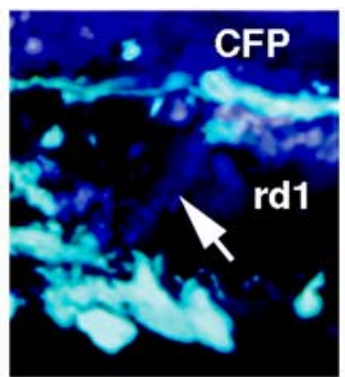

B
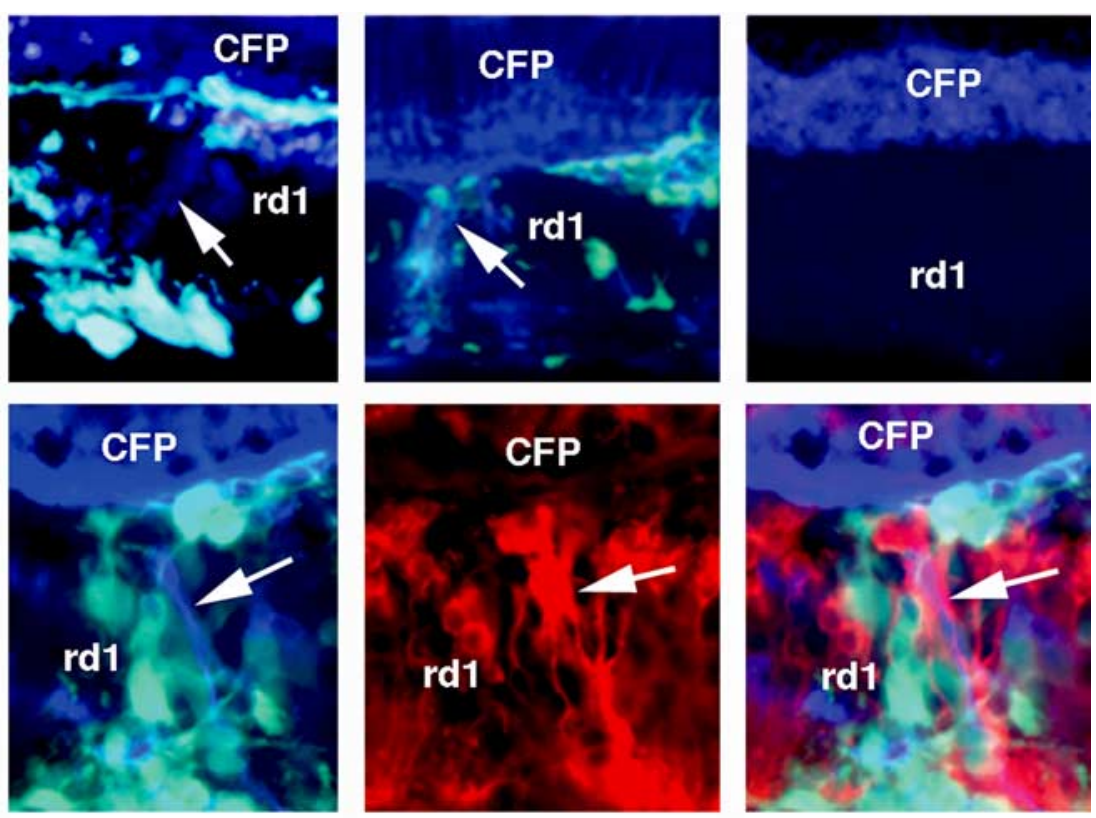

C

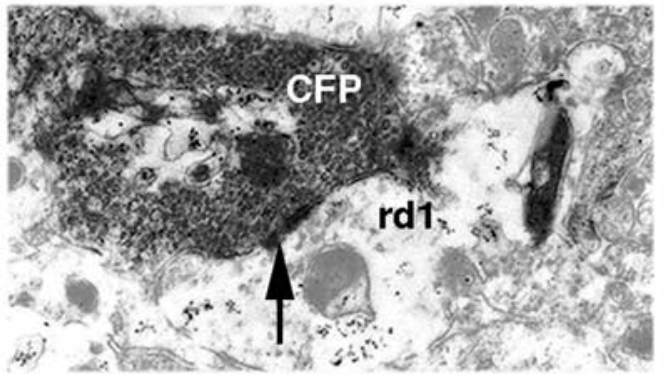

D

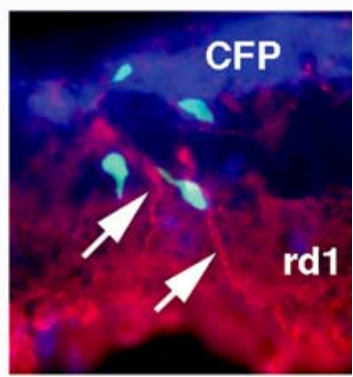

E

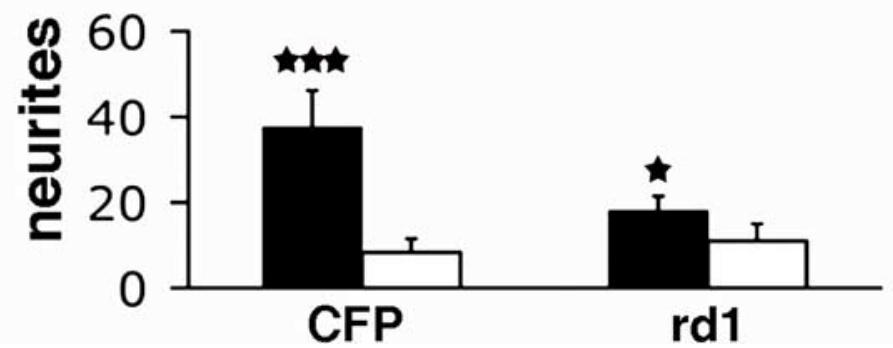

Figure 4. Engrafted progenitors promote neurite outgrowth in $r d 1$ retinas. $\boldsymbol{A}$, Cotransplantation of RPCs (GFP ${ }^{+}$; green) and neural retinal tissue (CFP ${ }^{+}$; blue) to the subretinal space of the $r d 1$ mouse resulted in an increased number of CFP ${ }^{+}$cells and processes (arrows) within the $r d 1$ retina. These fibers were accompanied by RPC migration (left) and were not seen in controls in which neural retina alone was transplanted (right), viewed at $30 \mathrm{~d}$ after surgery. Similar results were observed in the abutting retinal coculture system after $7 \mathrm{~d}$ in culture, in which CFP ${ }^{+}$neural retinas were placed adjacent to the outer surface of the $r d 1$ retina with NPCs at the interface (middle). $\boldsymbol{B}$, Triple labeling with CFP (blue), GFP (green), and PKC immunostaining (red) shows $\mathrm{CFP}^{+}$neurites (arrows) that originated from the grafted retina, coexpressed PKC, and extend into the synaptic layers of the $r d 1$ retina. C, CFP-immunolabeled neurites (CFP) formed symmetrical synapses (arrows) with unlabeled dendrites ( $r d 1$ ) in the $r d 1$ retina. $\boldsymbol{D}$, Neurites (arrows) expressing neurofilament (red), but not (FP, projected from the $r d 1$ retina across the graft-host border. $\boldsymbol{E}$, Quantification of neurites crossing the graft-host border originating from CFP ${ }^{+}$and $r d 1$ retinas. The number of neurites was significantly larger in $r d 1$ retinas engrafted with RPCs than those engrafted with neural retinal tissue $\left(n=6\right.$; ${ }^{*} p<$ $\left.0.05,{ }^{* * *} p<0.001\right)$. Scale bars: $\boldsymbol{A}, \boldsymbol{B}, \boldsymbol{D}, 30 \mu \mathrm{m} ; \boldsymbol{C}, 0.1 \mu \mathrm{m}$.

(i.e., progenitor cells, the nondystrophic retina, and the dystrophic retina). This was made possible by the availability of transgenic mice expressing different fluorescent proteins. To identify engrafted cells in $r d 1$ retina, we used nondystrophic P0 retinas from mice expressing an enhanced CFP transgene and progenitor cells isolated from mice expressing an enhanced GFP transgene.
Therefore, the RPCs exhibited green fluorescence, the $\mathrm{P} 0$ nondystrophic retinas exhibited blue fluorescence, and the dystrophic $r d 1$ retinas displayed no fluorescence. In this way, a clear border between $\mathrm{CFP}^{+}$ and $r d 1$ retinas could be readily distinguished (Fig. 4A, B,D).

One month after transplantation, a significantly larger number of $\mathrm{CFP}^{+}$cells and fibers appeared in the $r d 1$ retina when accompanied by migrating RPCs compared with the controls without added progenitor cells (Fig. 4A). Many $\mathrm{CFP}^{+}$ profiles in $r d 1$ retinas coexpressed neuronal markers, including amacrine and horizontal cell markers (calretinin and calbindin), a bipolar marker (PKC), and a ganglion cell maker (neurofilament) (Fig. $4 B)$. In a few specimens, it was possible to follow $\mathrm{CFP}^{+}$neurites projecting to the synaptic layers of the abutting $r d 1$ retina (Fig. $4 B$ ). The majority of RPCs present in $r d 1$ retinas had large somas with short or no processes, indicating a lack of morphological differentiation (Fig. $4 A, B, D$ ). Consistent with their morphology, few of the RPCs that had migrated expressed mature neuronal markers.

Immunoelectron microscopy demonstrated that cells and processes labeled with anti-GFP immunoparticles were present in the $r d 1$ retina and that conventional synapses between labeled and unlabeled processes were plentiful in the inner plexiform layer (Fig. 4C). Because the antibody against GFP recognizes both GFP and CFP proteins, we sought to clarify whether these synapses represented contacts between the $\mathrm{CFP}^{+}$nondystrophic $\mathrm{P} 0$ retinal grafts and $r d 1$ retinas or between $\mathrm{GFP}^{+} \mathrm{RPCs}$ and the $r d 1$ retina. For the in vitro experiments, we approached this question by substituting RPCs isolated from non-GFP mice at the interface of abutting retinas. After $7 \mathrm{~d}$ in culture, labeled synaptic contacts were again found in the $r d 1$ retina, similar to the previous results, suggesting that contacts between the abutting retinas are more prevalent than any potential contacts between RPCs [here GFP-negative $\left(\mathrm{GFP}^{-}\right)$] and the $r d 1$ retina. An alternate possibility, however, is that those synapses found in the $r d 1$ retina might be formed between migrating $\mathrm{CFP}^{+}$cells and the GFP ${ }^{-}$RPCs. To evaluate this possibility, we placed $\mathrm{GFP}^{+}$ RPCs at the interface between abutting nonfluorescent retinas from normal (B6) and $r d 1$ mice. In these cases, GFP ${ }^{+}$cells were not found to make synaptic contacts with unlabeled cells, confirming that the synapses between labeled and unlabeled profiles seen in the initial experiment were between the $\mathrm{CFP}^{+}$nondystrophic retina and the $r d 1$ retina (data not shown).

Using retinal neuronal markers to visualize neurites in the $r d 1$ 
retina, we tracked colabeled fibers from the $\mathrm{CFP}^{+}$retina (Fig. $4 \mathrm{~B}$ ), as well as $\mathrm{CFP}^{-}$ neurites of $r d 1$ origin (Fig. $4 D$ ). In both in vivo and in vitro experiments, quantification of neuronal fibers crossing the border zone showed that cografting with RPCs led to a significant increase in the number of neurites crossing the graft host border from both the nondystrophic neural retina and the $r d 1$ retina (Fig. $4 E$ ).

\section{RPC-induced neural integration is associated with reduction in CD44 and neurocan}

A continuous band of CD44 and neurocan immunostaining was found along the outer surface of $r d 1$ retinas that were cocultured with $\mathrm{CFP}^{+}$neural retinas (CD44 labeling in Fig. 5A). When large neurospheres were placed between abutting explants, the majority of RPCs (or NPCs) remained at the interface and very few progenitor cells migrated into either the $\mathrm{CFP}^{+}$or $r d 1$ retinas. In these instances, the CD44- and neurocan-positive band appeared slightly thinner but remained continuous (CD44 labeling in Fig. $5 B)$. We did not detect any $\mathrm{CFP}^{+}$cells or processes crossing the border into $r d 1$ retinas. When small neurospheres were placed at the interface of the abutting retinas, many progenitor cells migrated into $r d 1$ retinas in association with reduced immunoreactivity for CD44 and neurocan. Interestingly, $\mathrm{CFP}^{+}$ cells and processes were identified in the $r d 1$ retina exclusively in areas in which gaps were present in the band of immunolabeling (CD44 labeling in Fig. 5C). Similar results were observed in cotransplantation experiments.

\section{Progenitor cell-induced neurite outgrowth is MMP2 dependent}

In the absence of progenitor cells, pretreatment of $r d 1$ retinas with recombinant MMP2 resulted in an increase in the number of $\mathrm{CFP}^{+}$cells and fibers growing into $r d 1$ retinas (Fig. 6A,B). Conditioned medium from cocultures of $\mathrm{CFP}^{+}$retina and $r d 1$ retina grown with progenitor cells (containing active MMP2) also increased the number of neurites crossing the border between abutting retinas. These neurites originated from both $\mathrm{CFP}^{+}$(Fig. 6C) and $r d 1$ retinas (Fig. 6D). Conditioned medium from RPCs alone (not containing MMP2) had no effect on migration or integration (Fig. $6 C, D$ ). Furthermore, addition of the MMP inhibitor GM6001 ( $N-[(2 R)$-2(hydroxamidocarbonylmethyl)-4-methylpantanoyl]-L-tryptophan methylamide) significantly reduced the cell migration and neurite outgrowth induced by conditioned medium from retinal cocultures with added RPCs (Fig. 6C,D). When we substituted $M M P 2^{-/-}$retinas for the $r d 1$ retina in the abutting retinal cocultures with added RPCs, very few RPCs migrated into the $M M P 2^{-1-}$ retina (Fig. $6 E)$. In these cases, CD44 and neurocan immunostaining again appeared as a continuous band along the interface of the abutting retinas (data not shown). The number of neurites crossing the border of the abutting retinas was significantly decreased (Fig.
CD44
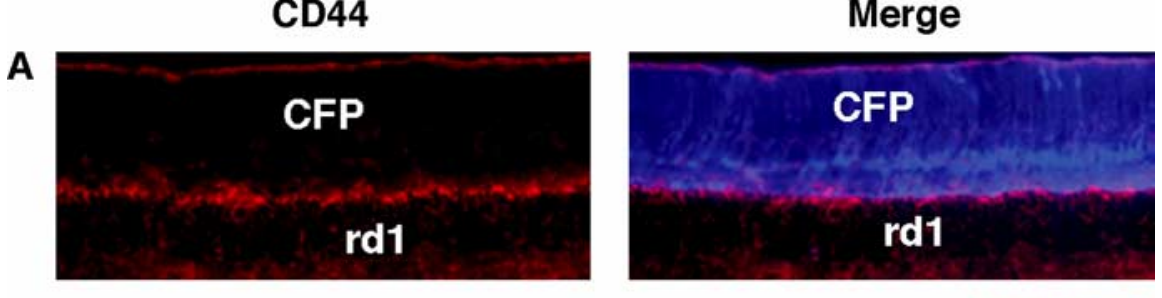

Merge
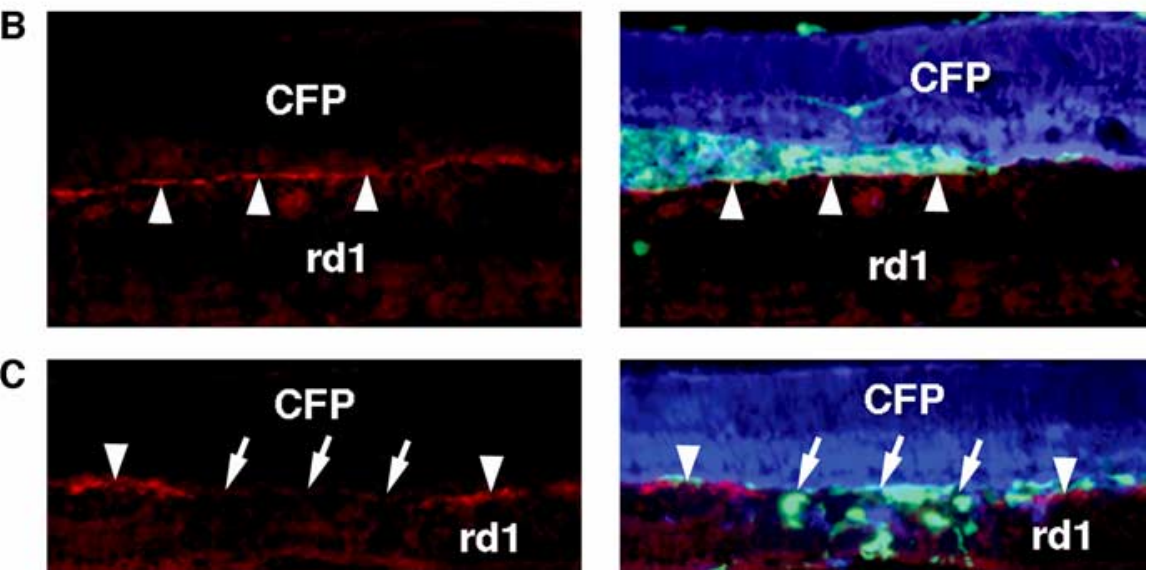

Figure 5. Neural integration between $\mathrm{CFP}^{+}$and $r d 1$ retinas correlated with a reduction of CD44 staining at the interface of abutting retinas. $A$, Without the addition of RPCS, CD44 immunolabeling (red) appeared as a continuous band at the interface of $\mathrm{CFP}^{+}$(blue) and $r d 1$ retinal explants. CFP ${ }^{+}$cellular profiles, including neurites, rarely crossed over the CD44 ${ }^{+}$band in the $r d 1$ ( into the $r d 1$ explant. C, When small RPC spheres were added, many progenitor cells migrated into the $r d 1$ explant, and CFP ${ }^{+}$ profiles appeared there as well. CFP ${ }^{+}$profiles crossed the interface exclusively in which gaps (arrows) were present in the band of CD44 immunolabeling (arrowheads). Scale bars, $30 \mu \mathrm{m}$.

$6 F)$ compared with controls $\left(\mathrm{CFP}^{+}\right.$retinas cocultured with wildtype retinas and RPCs).

\section{Discussion}

Recent studies of CNS stem and progenitor cell transplantation have revealed the remarkable potential of these cells in animal models of neurodegenerative diseases. However, translation of basic research into clinical therapies requires a better understanding of not only cell fate determination but also the ways in which donor cells interact with the host microenvironment. Here we have shown that exogenous CNS progenitor cells significantly enhance neurite outgrowth in the degenerating adult retina. The newly extended neurites originated from both immature (P0) nondystrophic retinal grafts/explants and mature (9 week) dystrophic $r d 1$ retinas, indicating that the permissive environment induced by progenitor cells favors not only developing neurites but also regenerative sprouting and synapse formation. Our results provide evidence that CNS progenitor cell-induced neurite outgrowth is MMP2 dependent. Although complex molecular interactions may be involved in the pathway by which this neurite outgrowth is promoted, the present study shows that activated MMP2 is essential. As such, this study also provides the first identification of one of the mechanisms by which progenitor cells alter the host environment.

MMP2 is known to play an important role in cell migration, neurite outgrowth, and synaptic plasticity during CNS development (Galko and Tessier-Lavigne, 2000; Webber et al., 2002) as well as in pathological processes (Duchossoy et al., 2001; Ahmed et al., 2005). It has been shown that treatment with MMP2 significantly increases extension of dorsal root ganglion neurites in 

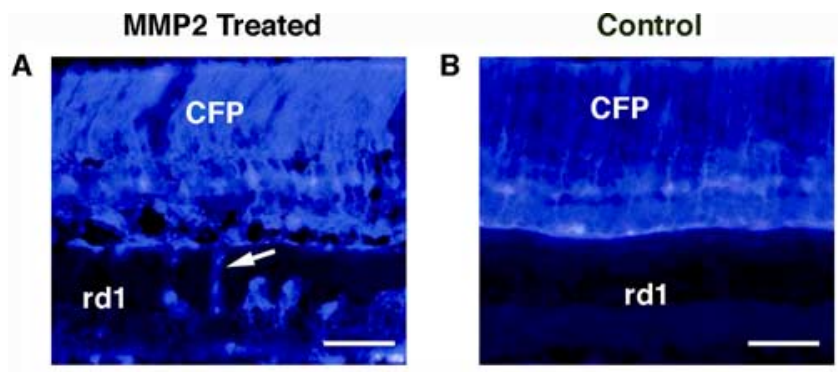

C

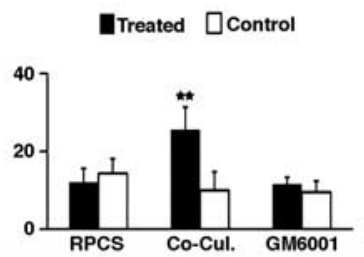

D

Ireated $\square$ Control

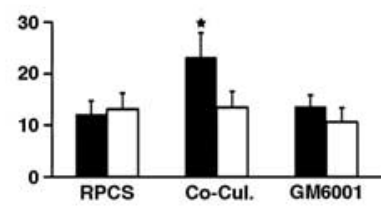

E

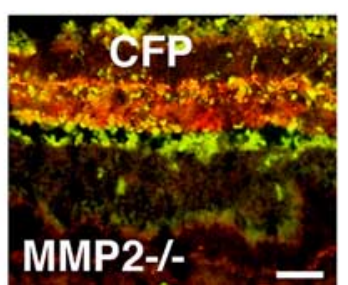

$\mathbf{F}$

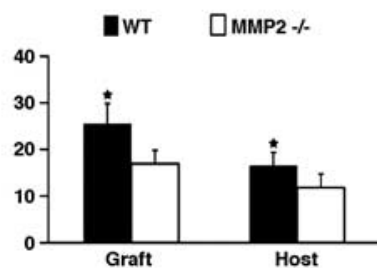

Figure 6. RPCs promoted MMP2-dependent neurite outgrowth in $r d 1$ retinal explants. $\boldsymbol{A}$ Pretreatment of $r d 1$ explants with recombinant MMP2 for $4 \mathrm{~h}$ before coculture with CFP ${ }^{+}$ explants led to an increased number of $\mathrm{CFP}^{+}$profiles (arrow) in the $r d 1$ retinas. $\boldsymbol{B}, \mathrm{CFP}^{+}$ profiles rarely appeared in $r d 1$ explants in control cocultures without MMP2 pretreatment. $C, D$, Quantification of neurites that coexpressed neuronal markers and CFP in rd1 explants ( $C$ and neurites that expressed neuronal markers and originated from $r d 1$ explants $(\boldsymbol{D})$ that crossed the interface. The number of neurites did not increase after treatment of abutting retinas with conditioned medium from RPCs, whereas it increased significantly after treatment with conditioned medium from cocultures ( $\mathrm{C} 0$-Cul.) of abutting retinas with added RPCs. This effect was blocked by addition of the MMP inhibitor GM6001 to explant cocultures ( $n=6$; ${ }^{*} p<0.05$, $\left.{ }^{* *} p<0.01\right) . E, C C^{+}$retinas cocultured with $M M P 2^{-/-}$retinas with $\mathrm{RPC}$ s added along the interface. Few RPCs migrated into $M M P 2^{-/-}$explants. $\boldsymbol{F}$, Quantification of neurites that crossed the border between abutting $\mathrm{CFP}^{+}$and either $M M P 2^{-/-}$or wild-type (WT) explants in the presence of RPCS. The number of CFP ${ }^{+}$neurites was significantly smaller in $M M P 2^{-1}$ explants compared with wild-type explants $\left(n=6\right.$; $\left.{ }^{*} p<0.05\right)$. Scale bars: $\boldsymbol{A}, \boldsymbol{B}, 30 \mu \mathrm{m} ; \boldsymbol{C}$, $5 \mu \mathrm{m}$.

the adult CNS (Ferguson and Muir, 2000). In addition, pharmacological inhibition of MMP2 and other metalloproteases by GM6001 leads to impaired outgrowth of retinal ganglion cell axons and their failure to enter targets (Webber et al., 2002).

Metalloprotease regulation of neurite outgrowth is complex because these enzymes participate in the proteolytic processing of a variety of ECM and cell surface components, many of which play a role in neurite extension and neural migration (Ray and Stetler-Stevenson, 1995; Giannelli et al., 1997; Zuo et al., 1998; Ferguson and Muir, 2000; Duchossoy et al., 2001). For example, MMPs degrade neurite-inhibiting chondroitin sulfate proteoglycans and lead to reduced scar formation, thereby permitting neurite extension into the adult sciatic nerve (Zuo et al., 1998), injured spinal cord (Duchossoy et al., 2001), and transected optic nerve (Ahmed et al., 2005). MMPs can also cleave and/or activate other substrates, including axon guidance molecules (Levi et al., 1996; Webber et al., 2002), growth factors, and cell adhesion molecules (Mechtersheimer et al., 2001; Kaczmarek et al., 2002), all of which can facilitate axon outgrowth in the developing CNS. Our data demonstrate that the enhancement of neurite out-

growth by RPCs is accompanied by lower expression of two neurite growth inhibitors, CD44 and neurocan. In addition, we show that RPC-induced neurite outgrowth is closely associated with MMP2 cleavage of these molecules, although we cannot exclude the possibility that degradation of other substrates may also be involved. Neurocan has been demonstrated to be a relevant MMP2 substrate (Turk et al., 2001). Our results suggest that both neurocan and CD44 can be specifically degraded by MMP2.

Several lines of evidence suggest that retinal pigment epithelial cells and Müller cells are the major sources of MMP2 in the retina under pathological conditions such as retinal degeneration (Padgett et al., 1997; Agapova et al., 2001; Limb et al., 2002). Release of MMP2 by Müller cells has been reported in culture (Limb et al., 2002) but not previously demonstrated in vivo. Moreover, few studies have investigated whether CNS progenitor cells can produce MMP2. One study reported that embryonic human CNS stem cells express MMP2 but that their expression is substantially decreased after differentiation (FrolichsthalSchoeller et al., 1999). We were unable to detect MMP2 expression or secretion by murine RPCs or NPCs, whereas we found highly upregulated expression in host $r d 1$ retinas after progenitor cell engraftment. In the present study, inhibiting Müller cell activity by DL- $\alpha$-aminoadipic acid treatment led to suppression of MMP2 induction. Our results thus strongly suggest that murine CNS progenitor cells do not produce MMP2, at least not at a detectable level, but instead stimulate secretion of this enzyme by host glial cells.

We found a positive correlation between the migration of progenitor cells into $r d 1$ host retinas and an enhancement of MMP2 expression, in part by Müller cells. This suggests that progenitor cell migration may be required to stimulate the latter to secrete MMP2. The mechanism by which glial secretion of MMP2 is induced has yet to be determined. It may reflect an overall activation of the glial cells during cell migration and/or a more specific response involving cell-cell contacts between the progenitor cells and host Müller cells, whereby activation of cellsurface receptors triggers signaling pathways, leading to MMP2 secretion.

During release, MMP2 can be activated by MMP14 present on the cell surface (d'Ortho et al., 1997; Overall and Lopez-Otin, 2002). MMP14 is expressed in the inner retina, but the distribution on specific cell types has not been delineated (Takano et al., 2005). Our results revealed expression of MMP14 by a majority of neurons in the $r d 1$ inner nuclear layer, as well as by some engrafted progenitor cells. These cells showed colocalization of gelatinase activity and MMP14, suggesting that MMP2 may be activated through its interaction with MMP14. Therefore, it is likely that pro-MMP2 released by Müller cells influences other cell types, including neurons, through activation by MMP14 expressed on those cells. It is important to note that MMP14 has also been shown to degrade CD44; therefore, we cannot exclude the fact that the presence of this molecule itself may play a direct role in CD44 digestion (Kajita et al., 2001). However, as we have shown in the $M M P 2^{-/-}$experiments, the presence of MMP14 alone is not sufficient to degrade CD44 or neurocan and thus, in the absence of MMP2 expression, does not have the ability to stimulate integration of grafted cells.

The concept that neural stem or progenitor cells are capable of rescuing imperiled host neurons has lead to the suggestion that these cells could be used for CNS repair in ways other than cell replacement (Ourednik et al., 2002). It has been proposed that the inherent developmental capacity of stem cells, such as their ability to secrete trophic and/or neuroprotective factors, persists 
after engraftment into the adult CNS, promoting neuronal survival and regeneration of the host environment (Imitola et al., 2004). In our study, despite intrinsic differences, both retina- and brain-derived progenitor cells were seen to have similar effects on neurite growth in the host retina. They did not themselves appear to produce MMP2 but induced MMP2 production by the host glial cells. Our findings therefore describe a novel mechanism by which grafted progenitor cells promote regeneration in the diseased CNS, in part through their interaction with resident glia wherein the latter are induced to express molecules that provide a permissive environment for neuronal regeneration. Thus, exogenous progenitor cells may be used not only for cell replacement and a source of neurotrophic support but also as a means to stimulate adult CNS plasticity by altering the host environment, allowing neural regeneration in the diseased brain, spinal cord, and retina. Progenitor cells from the retina and brain may therefore have a novel therapeutic role in the treatment of CNS degenerative diseases.

\section{References}

Agapova OA, Ricard CS, Salvador-Silva M, Hernandez MR (2001) Expression of matrix metalloproteinases and tissue inhibitors of metalloproteinases in human optic nerve head astrocytes. Glia 33:205-216.

Ahmed Z, Dent RG, Leadbeater WE, Smith C, Berry M, Logan A (2005) Matrix metalloproteases: degradation of the inhibitory environment of the transected optic nerve and the scar by regenerating axons. Mol Cell Neurosci 28:64-78.

Aramant RB, Seiler MJ, Ball SL (1999) Successful cotransplantation of intact sheets of fetal retina with retinal pigment epithelium. Invest Ophthalmol Vis Sci 40:1557-1564.

Asher RA, Morgenstern DA, Fidler PS, Adcock KH, Oohira A, Braistead JE, Levine JM, Margolis RU, Rogers JH, Fawcett JW (2000) Neurocan is upregulated in injured brain and in cytokine-treated astrocytes. J Neurosci 20:2427-2438.

Bradbury EJ, Moon LD, Popat RJ, King VR, Bennett GS, Patel PN, Fawcett JW, McMahon SB (2002) Chondroitinase ABC promotes functional recovery after spinal cord injury. Nature 416:636-640.

Costa S, Planchenault T, Charriere-Bertrand C, Mouchel Y, Fages C, Juliano S, Lefrancois T, Barlovatz-Meimon G, Tardy M (2002) Astroglial permissivity for neuritic outgrowth in neuron-astrocyte cocultures depends on regulation of laminin bioavailability. Glia 37:105-113.

d'Ortho MP, Will H, Atkinson S, Butler G, Messent A, Gavrilovic J, Smith B, Timpl R, Zardi L, Murphy G (1997) Membrane-type matrix metalloproteinases 1 and 2 exhibit broad-spectrum proteolytic capacities comparable to many matrix metalloproteinases. Eur J Biochem 250:751-757.

del Rio MR, DeFelipe J (1997) Synaptic connections of calretininimmunoreactive neurons in the human neocortex. J Neurosci 17:5143-5154.

Duchossoy Y, Horvat JC, Stettler O (2001) MMP-related gelatinase activity is strongly induced in scar tissue of injured adult spinal cord and forms pathways for ingrowing neurites. Mol Cell Neurosci 17:945-956.

Ferguson TA, Muir D (2000) MMP-2 and MMP-9 increase the neuritepromoting potential of schwann cell basal laminae and are upregulated in degenerated nerve. Mol Cell Neurosci 16:157-167.

Frolichsthal-Schoeller P, Vescovi AL, Krekoski CA, Murphy G, Edwards DR, Forsyth P (1999) Expression and modulation of matrix metalloproteinase-2 and tissue inhibitors of metalloproteinases in human embryonic CNS stem cells. NeuroReport 10:345-351.

Gaillard A, Gaillard F, Roger M (1998) Neocortical grafting to newborn and adult rats: developmental, anatomical and functional aspects. Adv Anat Embryol Cell Biol 148:1-86.

Galko MJ, Tessier-Lavigne M (2000) Function of an axonal chemoattractant modulated by metalloprotease activity. Science 289:1365-1367.

Ghosh F, Wong F, Johansson K, Bruun A, Petters RM (2004) Transplantation of full-thickness retina in the rhodopsin transgenic pig. Retina 24:98-109.

Giannelli G, Falk-Marzillier J, Schiraldi O, Stetler-Stevenson WG, Quaranta $\mathrm{V}$ (1997) Induction of cell migration by matrix metalloprotease- 2 cleavage of laminin-5. Science 277:225-228.

Imitola J, Park KI, Teng YD, Nisim S, Lachyankar M, Ourednik J, Mueller FJ,
Yiou R, Atala A, Sidman RL, Tuszynski M, Khoury SJ, Snyder EY (2004) Stem cells: cross-talk and developmental programs. Philos Trans R Soc Lond B Biol Sci 359:823-837.

Jablonski MM, Iannaccone A (2000) Targeted disruption of Muller cell metabolism induces photoreceptor dysmorphogenesis. Glia 32:192-204.

Kaczmarek L, Lapinska-Dzwonek J, Szymczak S (2002) Matrix metalloproteinases in the adult brain physiology: a link between c-Fos, AP-1 and remodeling of neuronal connections? EMBO J 21:6643-6648.

Kajita M, Itoh Y, Chiba T, Mori H, Okada A, Kinoh H, Seiki M (2001) Membrane-type 1 matrix metalloproteinase cleaves $\mathrm{CD} 44$ and promotes cell migration. J Cell Biol 153:893-904.

Kwan AS, Wang S, Lund RD (1999) Photoreceptor layer reconstruction in a rodent model of retinal degeneration. Exp Neurol 159:21-33.

Levi E, Fridman R, Miao HQ, Ma YS, Yayon A, Vlodavsky I (1996) Matrix metalloproteinase 2 releases active soluble ectodomain of fibroblast growth factor receptor 1. Proc Natl Acad Sci USA 93:7069-7074.

Limb GA, Daniels JT, Pleass R, Charteris DG, Luthert PJ, Khaw PT (2002) Differential expression of matrix metalloproteinases 2 and 9 by glial Muller cells: response to soluble and extracellular matrix-bound tumor necrosis factor-alpha. Am J Pathol 160:1847-1855.

Lu P, Jones LL, Snyder EY, Tuszynski MH (2003) Neural stem cells constitutively secrete neurotrophic factors and promote extensive host axonal growth after spinal cord injury. Exp Neurol 181:115-129.

McCawley LJ, Matrisian LM (2001) Matrix metalloproteinases: they're not just for matrix anymore! Curr Opin Cell Biol 13:534-540.

Mechtersheimer S, Gutwein P, Agmon-Levin N, Stoeck A, Oleszewski M, Riedle S, Postina R, Fahrenholz F, Fogel M, Lemmon V, Altevogt P (2001) Ectodomain shedding of L1 adhesion molecule promotes cell migration by autocrine binding to integrins. J Cell Biol 155:661-673.

Ogawa Y, Sawamoto K, Miyata T, Miyao S, Watanabe M, Nakamura M, Bregman BS, Koike M, Uchiyama Y, Toyama Y, Okano H (2002) Transplantation of in vitro-expanded fetal neural progenitor cells results in neurogenesis and functional recovery after spinal cord contusion injury in adult rats. J Neurosci Res 69:925-933.

Ourednik J, Ourednik V, Lynch WP, Schachner M, Snyder EY (2002) Neural stem cells display an inherent mechanism for rescuing dysfunctional neurons. Nat Biotechnol 20:1103-1110.

Overall CM, Lopez-Otin C (2002) Strategies for MMP inhibition in cancer: innovations for the post-trial era. Nat Rev Cancer 2:657-672.

Padgett LC, Lui GM, Werb Z, LaVail MM (1997) Matrix metalloproteinase-2 and tissue inhibitor of metalloproteinase- 1 in the retinal pigment epithelium and interphotoreceptor matrix: vectorial secretion and regulation. Exp Eye Res 64:927-938.

Ray JM, Stetler-Stevenson WG (1995) Gelatinase A activity directly modulates melanoma cell adhesion and spreading. EMBO J 14:908-917.

Takano A, Hirata A, Inomata Y, Kawaji T, Nakagawa K, Nagata S, Tanihara H (2005) Intravitreal plasmin injection activates endogenous matrix metalloproteinase-2 in rabbit and human vitreous. Am J Ophthalmol 140:654-660.

Teng YD, Lavik EB, Qu X, Park KI, Ourednik J, Zurakowski D, Langer R, Snyder EY (2002) Functional recovery following traumatic spinal cord injury mediated by a unique polymer scaffold seeded with neural stem cells. Proc Natl Acad Sci USA 99:3024-3029.

Turk BE, Huang LL, Piro ET, Cantley LC (2001) Determination of protease cleavage site motifs using mixture-based oriented peptide libraries. Nat Biotechnol 19:661-667.

Webber CA, Hocking JC, Yong VW, Stange CL, McFarlane S (2002) Metalloproteases and guidance of retinal axons in the developing visual system. J Neurosci 22:8091-8100.

Zhang Y, Sharma RK, Ehinger B, Perez MT (1999) Nitric oxide-producing cells project from retinal grafts to the inner plexiform layer of the host retina. Invest Ophthalmol Vis Sci 40:3062-3066.

Zhang Y, Caffe AR, Azadi S, van Veen T, Ehinger B, Perez MT (2003) Neuronal integration in an abutting-retinas culture system. Invest Ophthalmol Vis Sci 44:4936-4946.

Zhang Y, Kardaszewska AK, van Veen T, Rauch U, Perez MT (2004) Integration between abutting retinas: role of glial structures and associated molecules at the interface. Invest Ophthalmol Vis Sci 45:4440-4449.

Zuo J, Ferguson TA, Hernandez YJ, Stetler-Stevenson WG, Muir D (1998) Neuronal matrix metalloproteinase-2 degrades and inactivates a neuriteinhibiting chondroitin sulfate proteoglycan. J Neurosci 18:5203-5211. 